\title{
False-Positive Elevation of CK-MB Levels with Chest Pain in Lung Adenocarcinoma
}

\author{
Takayo Ota $^{a}$ Yoshikazu Hasegawa ${ }^{a}$ Eriko Muratab Noriko Tanakac \\ Masahiro Fukuoka ${ }^{a}$ \\ aDepartment of Medical Oncology, Izumi City General Hospital, Izumi, Japan; \\ ${ }^{b}$ Department of Cardiology, Izumi City General Hospital, Izumi, Japan; 'Department of \\ Radiology, Izumi City General Hospital, Izumi, Japan
}

\section{Keywords}

Chest pain $\cdot$ CK-MB $\cdot$ Lung adenocarcinoma

\begin{abstract}
The creatinine kinase (CK)-MB assay can be used for the early diagnosis of acute coronary syndrome. We describe the case of an 82-year-old male with lung adenocarcinoma who presented with chest pain. While laboratory findings showed elevated CK-MB levels, there was no cardiac injury. A chest computed tomography scan revealed pleural carcinomatosis. Later, electrophoretic analysis of CK showed a normal CK-MB range but increased CK-BB levels and the presence of macro CK type 2. We determined that the patient's chest pain originated from the visceral pleural invasion of lung cancer. Because of the methods used to measure the CK$\mathrm{MB}$ isozyme, the CK-MB level appeared elevated.

\section{Introduction}

Creatinine kinase (CK)-MB is a $\mathrm{CK}$ isozyme that exists mostly in the myocardium, but $5-7 \%$ of this isozyme exists in skeletal muscle [1]. CK-MB is a useful cardiac marker for acute myocardial infarction. However, with the introduction of troponin, which is more sensitive and accurate than CK-MB for detecting cardiac injury, the measurement of CK-MB is no longer required, and several guidelines state that CK-MB is not useful for the diagnosis of acute coronary syndrome $[2,3]$. We report a case of chest pain in a patient with lung adenocarcinoma in which the CK-MB level was increased, but cardiac injury was not confirmed. 


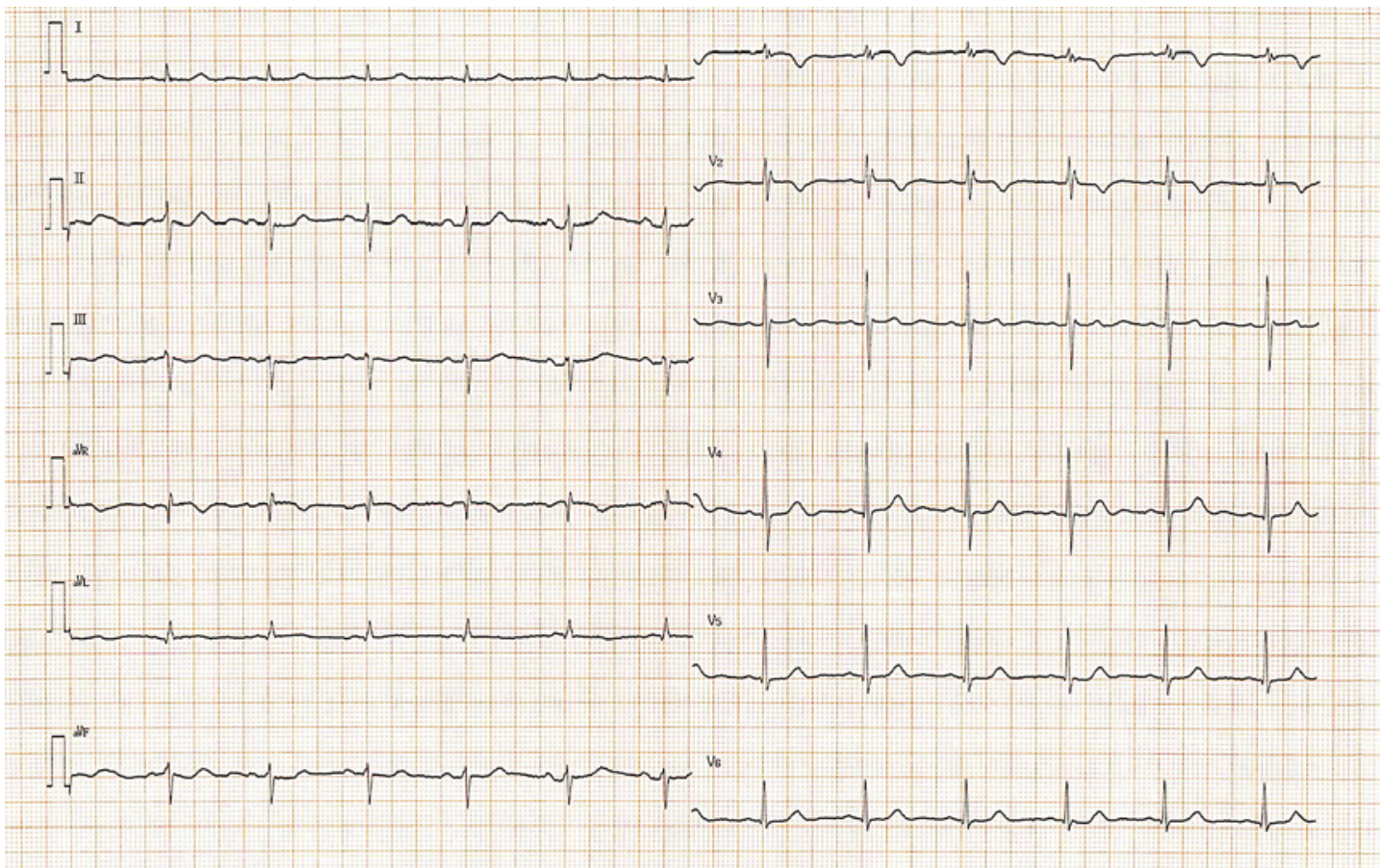

Fig. 1. ECG showing a normal sinus rhythm with no ST-segment changes.
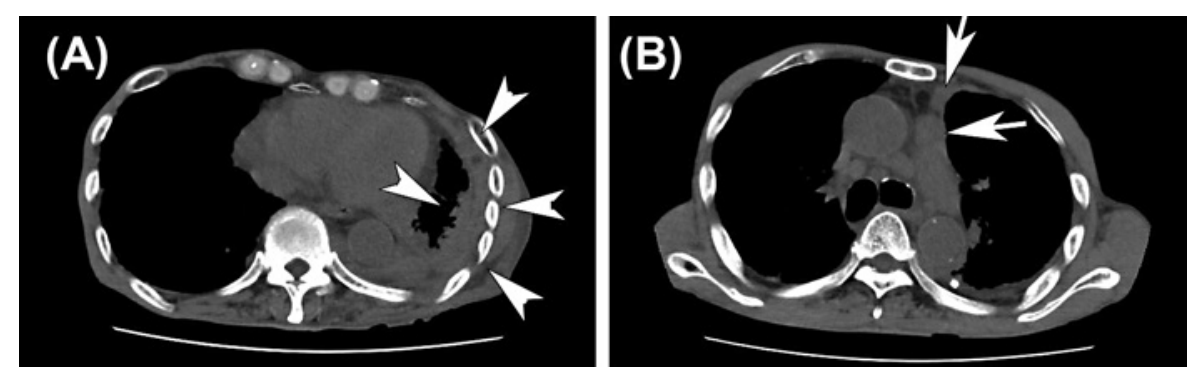

Fig. 2. Chest CT scan showing left pleural thickening (white arrowheads; $\mathbf{A}$ ) and enlarged lymph nodes (white arrows; B).

\section{Case Report}

An 82-year-old male with stage IV lung adenocarcinoma presented with worsening chest pain while lying in bed. He was hospitalized 2 days earlier because of general fatigue and loss of appetite after vinorelbine treatment $\left(25 \mathrm{mg} / \mathrm{m}^{2}\right)$, which had been administered as a secondline therapy 22 days before hospitalization.

He had been suffering from chest pain for 1 week without seeking any medical help. He had a similar episode of worsening chest pain in the sternum approximately $8 \mathrm{~h}$ earlier at night during his hospitalization stay. Measurement of his vital signs at that time showed a blood pressure of $128 / 77 \mathrm{~mm} \mathrm{Hg}$, a heart rate of 72 beats/min with a regular pulse, and a $\mathrm{SpO}_{2}$ value of $96 \%$. Because his vital signs were stable, his condition was monitored, and no further testing was performed. 
Fig. 3. Electrophoresis of serum CK showing increased CK-BB and macro CK type 2 levels, migrating cathodal to CK-MM (arrow).

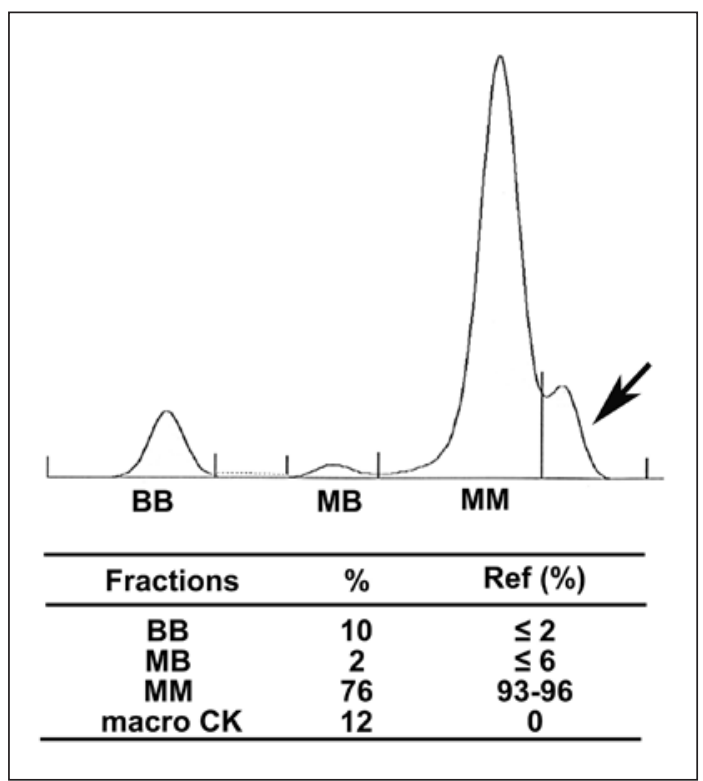

During the second episode of chest pain, measurement of his vital signs showed a blood pressure of $144 / 85 \mathrm{~mm} \mathrm{Hg}$, a heart rate of 68 beats/min with a regular pulse, and a $\mathrm{SpO}_{2}$ value of $96 \%$ when breathing room air. An immediate ECG showed no ST-segment changes (Fig. 1). A nitroglycerine tablet did not reduce the pain. Blood tests revealed increased total CK (547 U/L) and CK-MB (200 U/L) levels but normal levels of troponin I. An ECG showed neither hypokinesis nor akinesis of either ventricle. A chest computed tomography scan showed left pleural thickening and enlarged mediastinal lymph nodes (Fig. 2).

On day four after the second chest pain episode, his laboratory findings still showed elevated CK (579 U/L) and CK-MB (379 U/L) levels. Electrophoretic analysis of serum harvested on day four revealed that $2 \%$ of the total CK content was CK-MB, which was in the normal range, and CK-BB and macro CK type 2 levels were increased (Fig. 3). These results indicate that our patient's chest pain did not originate from cardiac injury but rather from lung adenocarcinoma metastasis. Subsequently, his chest pain was controlled by hydromorphone administration, as requested by the patient.

\section{Discussion}

CK is an enzyme involved in energy metabolism that catalyzes the phosphorylation of creatinine and adenosine triphosphate [1]. Cytosolic CK exists as a dimeric molecule with two subunits, $\mathrm{M}$ and $\mathrm{B}$. Three CK isozymes exist: CK-MM is expressed mainly in skeletal muscle, CK-MB is expressed primarily in the myocardium, and CK-BB is expressed mostly in the brain.

Moreover, CK is present as a higher-molecular-weight form, called macro CK [4], for which two types exist, type 1 and type 2 . Macro CK type 1 comprises a complex of CK isozymes and an immunoglobulin, while macro CK type 2 constitutes a mitochondrial CK polymer. Electrophoretically, macro CK type 1 is depicted as an anodal CK, migrating between CK-MM and CK-MB, and macro CK type 2 is depicted as a cathodic CK, migrating cathodal to CK-MB [5]. The prevalence of type 1 is $0.43-1.2 \%$, and that of type 2 is $0.5-3.7 \%[4,5]$. Macro CK type 1 is mostly found in patients with autoimmune diseases, and type 2 is found in severely ill patients, particularly in those with malignancies. While the presence of macro CK type 2 in 
lung cancer has been reported in the English literature, it is not stated whether these cases include lung adenocarcinoma $[6,7]$.

Abnormal serum levels of CK-BB are also often associated with neoplastic diseases. In lung cancer, increased serum CK-BB levels have been reported in cases of adenocarcinoma, small cell carcinoma, and squamous cell carcinoma [8,9]. The serum level of CK-BB is substantially increased in small cell carcinoma compared to that in other histological types of cancer [9]. The extent to which the serum CK-BB level increase is correlated with the number of metastatic sites, and a significant association exists between clinical response to therapies and serum CK-BB levels [10].

Increased levels of CK isozymes originate from tissues. Electrophoretic analyses of serum and lung cancer homogenate show the same patterns of CK-MB and CK-BB levels, indicating the tumor as the source of atypically increased CK isozyme levels [11].

In the present case, the laboratory findings showed increased serum CK and CK-MB levels. On the other hand, electrophoresis showed a standard range of CK-MB and elevated CK-BB and macro CK type 2 levels. CK-MB is measured by immuno-inhibition methods in which the $\mathrm{M}$ component is inactivated by an anti-M antibody, which leaves the $\mathrm{B}$ component active [12] (Cica Liquid CK-MB, Kanto Chemical Co., Inc.). By this method, after inactivating the $\mathrm{M}$ component, the remaining CK activity is doubled, which reflects a final CK-MB activity. CK-BB activity is thought to be initially inactive, and an anti-M antibody blocks CK-MM; the remaining CK activity is only from CK-MB. Since the M component is blocked, the CK-MB activity is halved. Hence, if some component is not able to be inactivated by anti-M antibodies, such as CK-BB or macro CK, the calculated CK-MB does not represent an accurate CK-MB activity.

In summary, we report the case of a patient with chest pain for whom the obtained results were confusing because of elevated CK-MB levels. The patient's chest pain did not originate from cardiac injury but rather from lung adenocarcinoma metastasis. When evaluating chest pain in malignancy, it is crucial to verify the origin, and not to rely on the CK-MB level only.

\section{Statement of Ethics}

The patient provided written informed consent for the publication of this case report and any accompanying images.

\section{Disclosure Statement}

We declare no conflicts of interest.

\section{Funding Sources}

No funding was received.

\section{Author Contributions}

T.0. drafted the manuscript. All authors were involved in the patient management and critically reviewed the manuscript. 


\section{References}

1 Cabaniss CD. Creatinine kinase. In: Walker HK, Hall WD, Hurst JW, editors. Clinical methods: the history, physical, and laboratory examinations. 3rd ed. Boston: Butterworths; 1990, pp. 161-3.

2 Amsterdam EA, Wenger NK, Brindis RG, Casey DE Jr, Ganiats TG, Holmes DR Jr, et al. 2014 AHA/ACC guideline for the management of patients with non-ST-elevation acute coronary syndromes: a report of the American College of Cardiology/American Heart Association Task Force on Practice Guidelines. J Am Coll Cardiol. 2014 Dec 23;64(24):e139-e228.

3 Roffi M, Patrono C, Collet JP, Mueller C, Valgimigli M, Andreotti F, et al. 2015 ESC guidelines for the management of acute coronary syndromes in patients presenting without persistent ST-segment elevation: task force for the management of acute coronary syndromes in patients presenting without persistent ST-segment elevation of the European Society of Cardiology (ESC). Eur Heart J. 2016 Jan 14;37(3):267-315.

4 Wiwanikit V. Macro creatine kinase. Chula Med J. 2003 Sep;47(9):601-11.

5 Galarraga B, Sinclair D, Fahie-Wilson MN, McCrae FC, Hull RG, Ledingham JM. A rare but important cause for a raised serum creatine kinase concentration: two case reports and a literature review. Rheumatology (Oxford). 2003 Jan;42(1):186-8.

6 Grobbel MA, Lawson NS, Calam RR. Cathodal creatine kinase band, a poor prognostic sign in malignancy. Clin Chem. 1982 Sep;28(9):1995-6.

7 McGing PG, Teeling M, McCann A, Kyne F, Carney DN. Non-M CK - a practical measure of creatine kinase isoenzymes in cancer patients. Clin Chim Acta. 1990 Mar 15;187(3):309-15.

8 Usui A, Fujita K, Imaizumi M, Abe T, Inoue K, Matumoto S, et al. Determination of creatine kinase isozymes in sera and tissues of patients with various lung carcinomas. Clin Chim Acta. 1987 Apr 15;164(1):47-53.

9 Arenas J, Diaz AE, Alcaide MJ, Santos I, Martínez A, Culebras JM. Serum CK-BB as a tumor marker in patients with carcinoma confirmed histologically. Clin Chim Acta. 1989 Jun 30;182(2):183-93.

10 Carney DN, Zweig MH, Ihde DC, Cohen MH, Makuch RW, Gazdar AF. Elevated serum creatine kinase BB levels in patients with small cell lung cancer. Cancer Res. 1984 Nov;44(11):5399-403.

11 Lee BI, Bach PM, Horton JD, Hickey TM, Davis WA. Elevated CK-MB and CK-BB in serum and tumor homogenate of a patient with lung cancer. Clin Cardiol. 1985 Apr;8(4):233-6.

12 Gerhardt W, Waldenström J. Creatine kinase B-subunit activity in serum after immunoinhibition of M-subunit activity. Clin Chem. 1979 Jul;25(7):1274-80. 